\title{
Exploration of physiological signals using different locomotion techniques in a VR adventure game
}

\author{
Stanislava Rangelova ${ }^{1,2}$, Simon Flutura ${ }^{2}$, Tobias Huber ${ }^{2}$, Daniel Motus ${ }^{1}$, and \\ Elisabeth André2 \\ 1 BMW Group, Munich, Germany \\ \{stanislava.rangelova, daniel.motus\}@bmw.de \\ 2 Human Centered Multimedia, Augsburg University, Augsburg, Germany \\ \{simon.flutura, andre\}@informatik.uni-augsburg.de \\ huber@hcm-lab.de
}

\begin{abstract}
In the past few years, Virtual reality gained popularity thanks to advancing technology. Although the consumer head-mounted displays provide affordable access to immersive virtual environments, one of the disadvantages is the induced discomfort during or after exposure, called simulation sickness. Simulation sickness is a condition of physiological discomfort felt during or after exposure to any virtual environment. One of the possible factors inducing simulation sickness could be locomotion, the travel of the user through the virtual space from one point to another. The study presented in this paper aims to expand the knowledge on how the human body responds to different locomotion techniques in a virtual reality environment. A within-subjects design $(\mathrm{n}=9)$ was conducted to explore simulation sickness outbreak, sense of presence and physiological responses induced by free teleportation and indirect locomotion in a virtual reality adventure game. The results showed that participants experienced significantly less simulation sickness while using the free teleportation during the game when compared to indirect locomotion. These findings indicate that simulation sickness symptoms can be reduced using free teleportation in a virtual reality adventure game.
\end{abstract}

Keywords: Locomotion - Simulation sickness - Virtual reality · Physiological signals · Adventure game · Presence - Head-mounted display.

\section{Introduction}

Virtual reality (VR) environments have certain advantages when it comes to evaluating Human-Computer Interaction (HCI). Modern computation technology and sensors enable immersive testing frameworks which can be accessed using head-mounted displays (HMDs). Thus the interest in VR has been increased. The beginning of the second decade of the twenty-first century marked an intensive hardware and software jump for the computer technology. This jump has affected the VR technology as well. With improved hardware and software, 
VR is widely accepted by researchers and users alike. VR systems offer a safe and fully controlled high fidelity environment which can provide low-cost setups for studies. In other words, an environment can be created which is close to a realistic experience without the liability issues or high costs [39]. In contrast to those advantages, VR has disadvantages as well. One of them which concerns the VR experience and the user's well-being directly is simulation sickness (SiS). SiS is a form of motion sickness which is induced by virtual environments and is also referred to as VR sickness or cybersickness [20]. It is a condition of physiological discomfort felt during or after exposure to a virtual environment. According to Cue conflict theory, the discrepancy between visual and motion cues is one of the assumed reasons for SiS [25]. SiS symptoms such as general discomfort, eye strain and difficulty in concentrating are more likely to be experienced while a user is using a fully immersive virtual environment (e.g. HMD) [12]. For example, eye strain could be experienced due to the close distance between the eyes and the HMDs screen, which could also induce headache. Those symptoms, also known as oculomotor symptoms, are the primary difference between SiS and motion sickness, where the nausea discomfort prevails $[15,14,37,6]$. The Ecological theory states that persons get sick from a prolonged postural instability during travel [26]. Locomotion, the travel from one point to another, could be one of the factors inducing SiS in VR simulations. Therefore, researchers have investigated which type of locomotion is suitable for VR games and what effect these types of locomotion have on the user [18,31]. The used measures are questionnaires and interviews with the users; however, physiological data were not collected. In this paper, a study which compares two different types of locomotion in a VR adventure game regarding SiS and presence is presented. In particular, physiological data and in-game events are reported as well as subjective data. The first type of locomotion is free locomotion which is known as point and teleport locomotion. The second one is inactive locomotion which mimics the controls of a standard game controller.

In the next section, related work will be provided. The methodology for the user study and the experimental setup will be described in Section 3, followed by the results of the trials. This paper concludes with a discussion of the results and shows a way toward future research.

\section{Related work}

With the growing body of VR research, the interest in locomotion in virtual environments has expanded as well. Thus, different locomotion techniques have been evaluated. Some of the techniques are: Omni-Directional Treadmill [7], redirected walking [24], walk-in-place [29], and teleportation [2]. An Omni-directional treadmill is a device similar to a treadmill used in the gyms which allows the user to walk in any direction in the virtual world $[30,11]$. These type of input devices are already on the market. Redirected walking allows the user to move through a large-scale VR area while physically stays in a much smaller workspace. This gives the opportunity to use VR in a reasonably small space while the simulation 
presents a different size of space. The virtual camera motion is manipulated in a way that the user's self-motion in the virtual world differs from the movements in the real world $[32,4]$. The walk-in-place technique allows the user to continuously walk in place while movements of the body are tracked and analyzed. The early implementations of this technique were based on processing of the head position using a neural network. More recent implementations track the legs position, below the knees, of the user to calculate the virtual locomotion $[36,35$, 41]. Teleportation is an instant movement of the user from one location to another in the virtual world $[8,3]$. Each of the listed techniques has its advantages and disadvantages which may also depend on the application's objectives.

In general, there are many different ways that a user can travel in VR using the controllers. Frommel et al. [9] investigated the effect of different controllerbased locomotion on the player experience in a VR game. In this paper, they pointed out that the most popular type of travels with controllers which are used in VR for entertainment purposes can be narrowed down to four:

- Free teleport locomotion

- Fixpoint Teleport Locomotion

- Indirect Locomotion

- Automatic Locomotion

The results from their studies showed that free teleport locomotion induced the lowest and the indirect locomotion induced the highest SiS scores. Therefore, in this paper, these two types of locomotion are evaluated in a VR adventure game. The free teleport locomotion is a locomotion technique which allows the user to teleport herself freely within the field of view. The user points to the desired direction and an arc is presented which ends with a point where the user's avatar will be teleported. In this paper, the free teleport locomotion will be referred to as free teleportation and additionally includes an avatar model which shows the location of the player after the teleportation. The indirect locomotion is a technique which allows the user to move around the virtual world using a similar input as a standard game controller. The user can move forward by pressing forward on a touchpad (HTC Vive Controller) or a joystick (Oculus Touch).

A recent study introduced a node-based locomotion technique which is using a predefined node position to which the user can travel with rapid, continuous, linear motion [10]. The technique was compared with indirect locomotion and free teleportation regarding SiS and presence. The results showed that the proposed node-based locomotion technique induced less SiS compared to the indirect locomotion. Both nausea and oculomotor clusters, based on the Simulation Sickness Questionnaire (SSQ) [13], were lower for node-based techniques compared to the indirect locomotion technique. However, the results stated that the mean score for nausea cluster of the free teleportation is slightly lower than the mean score of the node-based locomotion. Regarding presence, no differences were found between the conditions.

The two studies [9] and [10] compare different locomotion techniques regarding $\mathrm{SiS}$ in a virtual environment using subjective measurements. In this paper, 
a comparison of locomotion techniques using not only subjective measurements (questionnaires) but also objective measurements (physiological signals) is presented. These measurements give an essential insight into the users' physical response to the virtual world while traveling within the virtual world.

\section{Methods}

\subsection{Setup}

The system setup consisted of an HMD with a tracking system, controllers, sensors, and a computer for rendering the VR scene. The HMD was an HTC Vive with a field of view of $110^{\circ}$, a resolution of $1080 \times 1200$ pixels per eye, and a Dual AMOLED screen. The tracking system was a Vive Lighthouse system consisting of two black boxes which create a $360^{\circ}$ virtual space within which the position of the HMD and the controllers are tracked. The controllers are the standard controllers included in the package with the HMD. They have multiple input methods including grip buttons, a dual-stage trigger, and a touchpad. In the adventure game, the grip buttons were used as a trigger for grabbing objects in the virtual world. While the buttons are pressed the user holds the object, on the release of the button, the user drops the held object. The heart rate (HR), skin conductance level (SCL) and respiration rate (RR) signals were collected using Plux sensors [27]. All three signals were recorded with a frequency of 1 $\mathrm{kHz}$. The HR was collected using a blood volume pulse sensor attached to the tip of the ring finger on the left hand with velcro strips. The SCL was recorded through electrodermal activity sensors attached on the third and fourth fingers of the left hand. The RR was collected using piezoelectric respiration sensor attached to the abdomen via an elastic strapped belt. Then the data was sent to the data collection computer via Bluetooth and recorded by the Social Signals Interpretation (SSI) software developed by the University of Augsburg [38]. The experimental setup with the HMD and the Plux sensors is shown in Figure 1.

The SSI framework offers tools to record, analyze and recognize human behavior in real-time, such as gestures, mimics, head nods, and emotional speech. It supports streaming from multiple sensors and includes mechanisms for their synchronization. To integrate with other applications, SSI features a set of network plugins such as Transmission Control Protocol (TCP), User Datagram Protocol (UDP), User Datagram Protocol, OSC or Websockets. Beneath continuous streams, sporadic events can be recorded in synchronous. Particularly, SSI supports the machine learning pipeline in its full length (pre-processing, feature extraction, and online classification and fusion) and offers a graphical interface that assists a user to collect own training corpora and to obtain personalized models. It also suits the fusion of multimodal information at different stages including early and late fusion. SSI is written in $\mathrm{C}++$ and the source code is available under LGPL. All the data of the study is recorded by SSI which stored the recorded data in its own, simple, text-based data format. This way, the data of all sessions can later be further analyzed using different tools such as data analysis and machine learning features of SSI, Python or Matlab. 


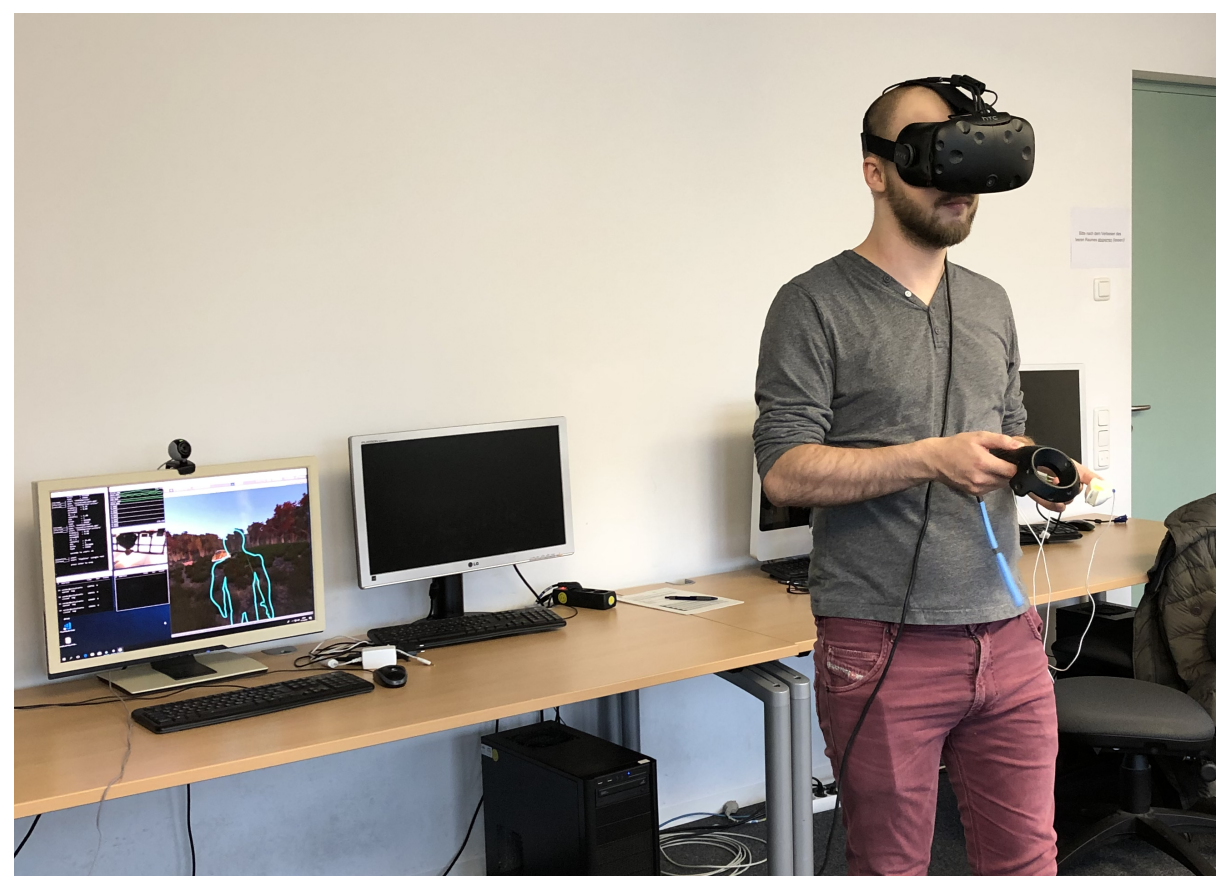

Fig. 1. A participant using the experimental setup. The controller is in his right hand while the sensors are attached to his left hand and chest. Behind him is the computer on which VR scene is rendering and the physiological signals are recording.

\subsection{Measures}

Blood volume pulse (BVP) is presented by changes in the volume of blood in the vessels. A non-invasive sensor can measure the BVP through light absorption of the skin and tissues and their level of illumination [1]. Usually, the sensor is attached to the non-dominant hand on the tip of the ring finger. From the BVP signal, the HR is calculated by estimating the time interval between the heartbeats, named the interbeat interval measured in seconds [23]:

$$
H R=60 \text { seconds/interbeatinterval }
$$

Prior studies displayed that immersion in a virtual world increased the HR with prolonged stay in the VR [33] and had a positive relationship with SiS onset [21].

Electrodermal activity (EDA) is a property of the human body that causes continuous variation in the electrical characteristics of the skin. It is measured with sensors which detect the changes in the passing a neglected amount of currency through the skin and the unit is micro-Siemens (uS). There are two 
main parts of the EDA which can be used to measure the ongoing electrodermal changes. The first one is the tonic level which is related to the background characteristics of the signal and a slower acting change. The second one is the phasic level which is associated with faster changes in the signal. EDA can be measured through the galvanic skin response (GSR), the electrodermal response, the psychogalvanic reflex, the skin conductance response, the sympathetic skin response, and the SCL. Most often the phasic response is used to present the results of the EDA data. However, this is just a part of the EDA complex. Typically the EDA changes when people are under stress which is shown by sweating on the inner side of the hand, the palms and the fingers. Previous research showed a connection between the SiS onset and SCL [19].

Respiration is the process of breathing in (inhalation) and breathing out (exhalation) [34]. The respiratory effort is measured by the number of breaths taken under a certain amount of time, often referred to us as an RR. Frequently this time is measured in minutes and therefore, the RR is given in breaths per minute which can be calculated into a frequency. A higher RR is related to arousal [19]. Furthermore, SiS scores had a significant positive correlation with respiration rate. The higher a participant's $\mathrm{RR}$ changes from the baseline, the more the participant reported SiS symptoms [16].

In another study, the RR was used as an objective measurement as well as GSR, HR, and skin temperature, in a flight simulator study comparing the presence in HMD and conventional setups [40]. There was no significant difference in the HR or RR. However, the skin resistance displayed more reactance in HMD condition.

Pre-questionnaire is a self-report instrument in which the participants are asked to answer questions to determine the socio-demographic information, previous gaming and VR experience. The questionnaire consists of seven questions, of which the first three questions were standard socio-demographic (age, gender, and education). The next two questions measured the previous gaming and HMD experience by using the five-point Likert scale (i.e., from strongly agree to strongly disagree). The last two questions checked whether the participants drank coffee or if they are smokers.

Simulation sickness questionnaire (SSQ) was provided to collect information about the participants state of well-being after the experiment. The questionnaire was originally developed by Kennedy and his colleagues in 1993 [13]. It is widely used to measure SiS and was the most suitable instrument for this project. The questionnaire consists of 16 questions where each question has four possible answers e.g. none, slight, average and severe. Each answer reflected the current condition of the participant and at what level each symptom is experienced. Each answer is scored with 0 - none, 1 - slight, 2 - moderate and 3 severe scores and corresponds to one of the three clusters (Nausea, Oculomotor, 
and Disorientation). Some of the symptoms such as general discomfort, difficulty focusing, difficulty concentrating, and blurred vision are part of two clusters.

iGroup Presence Questionnaire is a self-report instrument, which was used to gather information about the users VR experience and enjoyment [28]. It consists of 16 questions where each question could be answered once through a five-point Likert scale (from strongly agree to strongly disagree). The IPQ is based on a presence questionnaire originally developed by Witmer and Singer [42]. A few questions regarding enjoyment [17] were included in the IPQ questionnaire to measure the users emotional reaction to the VR game.

In-game events are events which were created to track the interactions of the participants with the virtual environment. Some of the events are related to the interaction with the inventory such as "Drop *item name* into inventory" (see Table 1). Others are related to more active interactions with objects (e.g. lockpick, lighter, glass bottle) such as "Pick up *item name*," "Drop *item name*" and "Chop wood." Furthermore, there are events which track the interaction with environmental objects like a car trunk or a light switch. At last, some events to monitor the position of the participant in the virtual environment were added like "Entered water area" or "Spawning to *spawn location*." All the events are recorded through SSI and can be further analyzed together with the synchronized physiological signals.

Table 1. List of the in-game events and their short description which were using during the VR session.

\begin{tabular}{|l|l|}
\hline Events & Description \\
\hline Pick up *item name* & The user picks up an object. \\
Drop *item name* & The user drops the picked object. \\
Drop *item name* into inventory & The user drops an object in the inventory bag. \\
Interact with *object* & The user interacts with an environmental object. \\
Unlock & The user unlocks the shack's door. \\
Chop wood & The user uses an ax to chop wood. \\
Adding wood & The user adds wood to the fire. \\
Enter Area & The user enters a particular area. \\
Spawning to *spawn location* & The user spawns at the given location. \\
\hline
\end{tabular}

\subsection{Participants}

Nine participants aged between 24 and 32 years $(\mathrm{M}=26.44 ; \mathrm{SD}=2.51)$ took part in the study. Only three female participated and therefore, gender as a variable was unequally distributed and could not be evaluated. The participants were students or employees of the University of Augsburg, Germany. Five of 
the participants described themselves as frequent video gamers who play computer or console video games. In respect to previous experience with HMDs, three participants had used an HMD before this trial, one of those participants experienced dizziness during previous VR session.

\subsection{Procedure}

An adventure exploratory VR game study with a within-subjects design was conducted. Two conditions were presented: free teleportation and indirect locomotion. Each participant took part in both conditions. The experimental design was counterbalanced by alternating the starting condition for each participant. Before starting the VR session, the participants were informed of the procedure and the aim of the study. A consent form was handed to each participant followed by the pre-questionnaire. After that, instructions about the game controls were given and the Plux sensors were attached to the body. With the help of the researcher, the HMD was put on the head. For the first one minute, the participants were instructed not to move, only to rotate the head if he wanted to. During that time baseline data was collected. The game started with the participants spawning in a forest. There they were instructed to walk around and to find a car or a fireplace, whichever she found first (fig. 2). At the car's location, the participants were able to interact with the car's trunk to open it. Inside of the trunk, there were a few lockpicks and a lighter laying around. These objects could be picked up and stored in the participants' inventory for later usage. After the interaction with the car, the trunk could be closed or left open.

When the participants arrived at the fireplace, they were told to light a fire. To do that they had to grab logs of wood which were placed around the fireplace and had to throw them into the smoldering fire. For each piece of wood, the fire grew larger. There was also an ax placed close to the fireplace. The participants could use this ax to cut the logs into smaller pieces of wood which were also usable in the fire. The aim of this interaction was to make a big fire by using as many pieces of wood as the participant wanted to but always at least three pieces.

After lighting the fire, the participants were instructed to head to a shack located not far away. On the way to the shack, they had to cross a small river by using a bridge or by going the through the water. The shack door was locked, so the participants had to use a lockpick to unlock the door. If they did not pick up the lockpick previously, they had to go back to the car and get a lockpick from the car trunk.

After entering the shack, the participants were able to use the lighter or a light switch to brighten up the dark shack. Here, the participants were free to explore the room and to interact with various objects placed inside of the shack. After that, the participants opened the door to leave the shack and the game was over. All sensors, the HMD, and the controller were removed. Before the session was over, the IPQ and SSQ were given to the participants. 

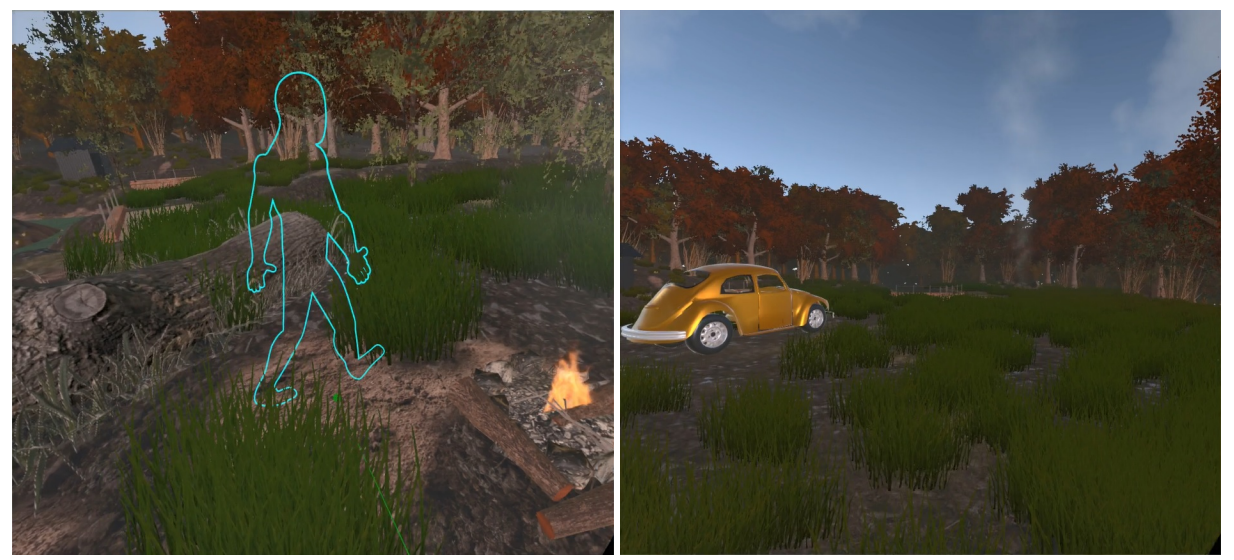

Fig. 2. Two different experimental conditions: free teleportation (on the left side) and indirect locomotion (on the right side).

\section{Results}

The results showed that there were differences between the HR and SCL baseline and the ones recorded during the game. The participants felt more nauseous and disorientated during the indirect locomotion. However, there were no differences regarding presence and physiological signals between the conditions. The software toolbox used to analyze the data was Biosppy, which is written in Python $3[5]$.

The results of the SSQ indicated that there was a significant difference between the free teleportation and indirect locomotion conditions regarding SiS. Paired sample t-test revealed statistically significant differences between the two conditions in Nausea cluster (free teleportation: $\mathrm{M}=9.54 ; \mathrm{SD}=10.67$, indirect locomotion: $\mathrm{M}=29.68 ; \mathrm{SD}=19.34 ; \mathrm{t}(8)=-3.59 ; \mathrm{p}=0.007)$, Disorientation cluster (free teleportation: $\mathrm{M}=41.76 ; \mathrm{SD}=28.70$, indirect locomotion: $\mathrm{M}=$ $75.79 ; \mathrm{SD}=49.27 ; \mathrm{t}(8)=-2.63 ; \mathrm{p}=0.030$ ), Total score (free teleportation: $\mathrm{M}$ $=298.96 ; \mathrm{SD}=215.25$, indirect locomotion: $\mathrm{M}=548.79 ; \mathrm{SD}=309.60 ; \mathrm{t}(8)=$ $-3.85 ; \mathrm{p}=0.005)$. These results suggested that free teleportation really had an effect on SiS symptoms from the Nausea and Disorientation clusters and Total score. Specifically, our results suggested that when participants use free teleportation, the SiS onset decreases. The same test revealed not significant difference between the two condition but a trend in Oculomotor cluster (free teleportation: $\mathrm{M}=28.64 ; \mathrm{SD}=24.50$, indirect locomotion: $\mathrm{M}=41.27 ; \mathrm{SD}=29.14 ; \mathrm{t}(8)=$ $-2.18 ; \mathrm{p}=0.06)$. These results implied that the type of locomotion technique had no effect on SiS symptoms from the Oculomotor cluster. One of the participants stopped the session due to severe discomfort while using the indirect locomotion. The most rated symptoms for the free teleportation condition were "Difficulty focusing," "Blurred vision," and "Eyestrain." For the other condition, Indirect locomotion, they were "General discomfort," "Difficulty focusing," and "Blurred 
vision." The overall score indicated that the participants felt less discomfort using the point and teleport locomotion technique (fig. 3).

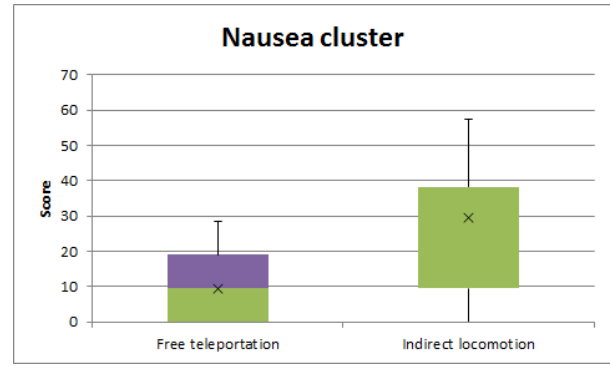

(a)

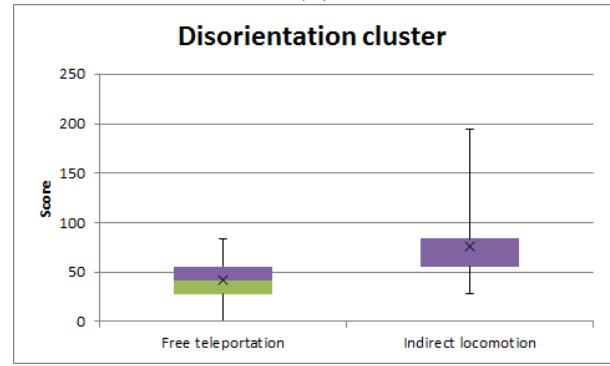

(c)

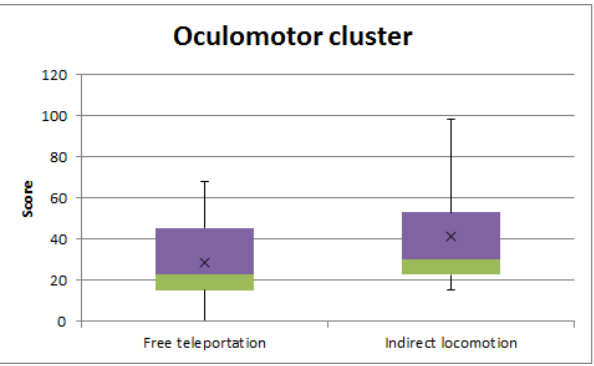

(b)

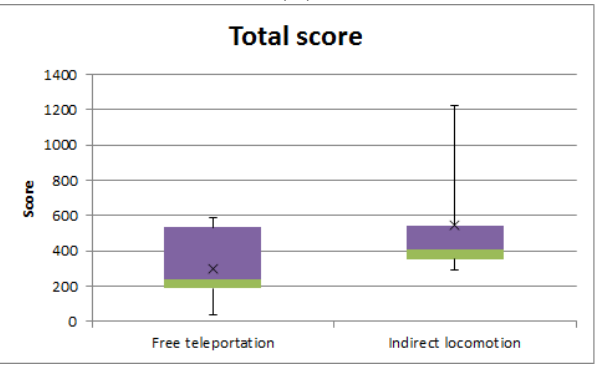

(d)

Fig. 3. Nausea (a), Oculomotor (b) and Disorientation (c) clusters as well as Total score $(\mathrm{d})$ for free teleportation and indirect locomotion conditions.

Regarding the sense of presence, there were no significant differences between the free teleportation and indirect locomotion. However, the indirect locomotion was rated slightly higher on the items "In the computer-generated world I had a sense of "being there."; "I felt like I was just perceiving pictures."; "I still paid attention to the real environment." On the items regarding enjoyment in the VR game, the participants gave positive responses for both conditions which did not yield any differences. The participants with previous gaming experience reported a higher sense of presence.

A paired sample t-test revealed no statistically significant difference between the two conditions in the HR (free teleportation: $\mathrm{M}=90 ; \mathrm{SD}=11.59$, indirect locomotion: $\mathrm{M}=95 ; \mathrm{SD}=14.16 ; \mathrm{t}(8)=-1.34 ; \mathrm{p}=0.218$ ); $\mathrm{SCL}$ (free teleportation: $\mathrm{M}=0.48 ; \mathrm{SD}=0.44$, indirect locomotion: $\mathrm{M}=0.59 ; \mathrm{SD}=0.83 ; \mathrm{t}(8)=$ $-0.41 ; \mathrm{p}=0.689$ ); $\mathrm{RR}$ (free teleportation: $\mathrm{M}=0.23 ; \mathrm{SD}=0.03$, indirect locomotion: $\mathrm{M}=0.23 ; \mathrm{SD}=0.01 ; \mathrm{t}(8)=0.22 ; \mathrm{p}=0.83)$. The HR shows a trend towards reduced $\mathrm{HR}$ in the free teleportation condition. That means that participants had a lower HR. The results are shown in Figure 4.

However, a comparison between the baseline HR recorded before the game and during the VR game HR revealed a significant difference for the free tele- 
portation condition (VR game: $\mathrm{M}=98 ; \mathrm{SD}=7.23$, baseline: $\mathrm{M}=90 ; \mathrm{SD}=$ $11.6 ; \mathrm{t}(8)=2.45 ; \mathrm{p}=0.04)$. Significant difference was found as well between the baseline and during the VR game SCL for the free teleportation condition (VR game: $\mathrm{M}=0.78 ; \mathrm{SD}=0.74$, baseline: $\mathrm{M}=0.48 ; \mathrm{SD}=0.44 ; \mathrm{t}(8)=0.25 ; \mathrm{p}=$ 0.039 ). The same analysis showed no significant difference for the baseline and the VR game $\mathrm{RR}$ for the free teleportation (VR game: $\mathrm{M}=0.23 ; \mathrm{SD}=0.01$, baseline: $\mathrm{M}=0.23 ; \mathrm{SD}=0.03 ; \mathrm{t}(8)=-0.6 ; \mathrm{p}=0.57)$.

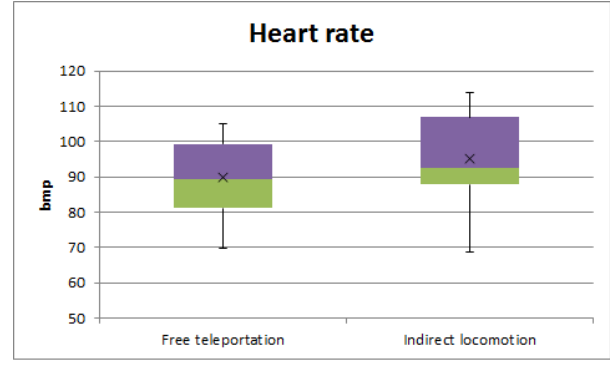

(a)

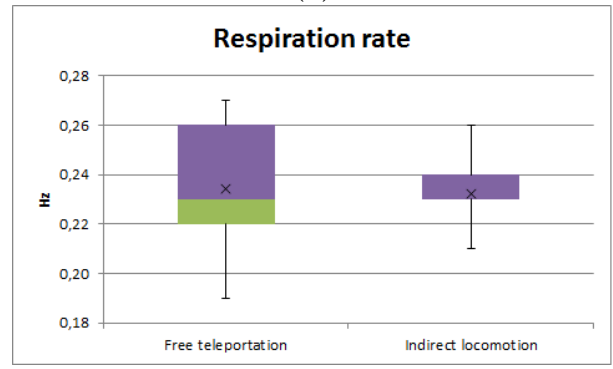

(c)

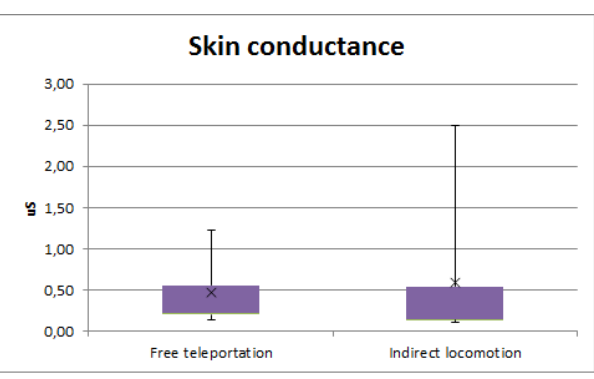

(b)

Fig. 4. Heart rate (a), skin conductance (b) and respiration rate (c) for free teleportation and indirect locomotion conditions.

The most recorded in-game events were "Drop *item name*," "Drop *item name* into inventory," and "Chop wood." Due to personal preferences, each participant was more involved with one or another from the events listed above and therefore an overview analysis was conducted. Nevertheless, further analysis is required in order to show whether a correlation between the physiological signals and certain events exists or not.

\section{Discussion}

The popularity of HMDs among users and researchers has made this medium a valuable evaluation tool in HCI research. However, the technology of VR and in particular HMDs has its disadvantage regarding users' well-being, namely SiS. 
Prior research has documented the positive correlation between locomotion and SiS onset in VR. Habgood et al. [10], for example, reported that participants felt less SiS during locomotion techniques using rapid continues movements between nodes. However, these studies were evaluating the locomotion techniques via subjective measures such as questionnaires. In this paper two different locomotion techniques, free teleportation and indirect locomotion were tested in a VR adventure game using objective (physiological signals) and subjective (questionnaires) measurements.

The questionnaire results showed a significant difference in SiS outbreak. In particular, participants reported higher SSQ scores in Nausea and Disorientation clusters and total score. These results provide evidence to support earlier studies that there are differences between the free teleportation and indirect locomotion regarding SiS. Moreover, the results align with previously conducted studies on locomotion techniques [9]. Two of the most induced symptoms, "Difficulty focusing" and "Blurred vision," were the same for both conditions. A possible reason could be the graphics quality of the VR game and the screen of the HMDs. Even though all the participants were instructed to adjust the distance between the lenses for a sharper view, some of them did not perceive any change regarding the sharpness after using the adjustment button on the HMD. Another symptom, "Eyestrain," was mostly felt during the free locomotion condition. That could be explained with a gaze following and focusing on the avatar during the process of teleportation.

An interesting observation was that the participants with previous gaming experience, felt more comfortable using any locomotion technique compare to the participants without gaming experience, who preferred to walk. Some of the participants commented that when they were looking down to pick up an object from the ground or to drop an object into the inventory bag, they felt severe eyestrain and dizziness. A possible explanation could be the fact that those participants had no previous gaming experience and therefore, they were not used to rapid changes in the field of view which is a common aspect of many video games.

Regarding the presence and enjoyment during the VR adventure game, no significant difference was found between the free teleportation and indirect locomotion conditions. The participants experienced the same level of presence regardless of the locomotion technique. These findings are consistent with those of Habgood et al. [10] who found no difference comparing three locomotion techniques regarding presence. Furthermore, the participants reported a slightly higher presence in the indirect locomotion. A possible explanation for that could be that this technique is closer to the real world walking. Surprisingly, the participants with the highest SiS score reported that they felt present and had a sense of being in the virtual world. These results did not support a previous research [22] on presence which stated that a lower sense of presence might increase the SiS onset.

The slight decrease in the HR corresponds to the lower score in the SSQ for the free teleportation. One possible explanation of why the signals did not show 
a greater difference is that the participants had to move in order to interact with the VR environment. This could bring noise to the signal and later is more difficult to compare the signals. The significant difference comparing baseline and VR game data, showed in the previous section, might be due to the participants' movements during the game which can increase the HR and the SCL. In other words, the participants did more movements with the free teleportation than with indirect locomotion. These results differ from previous research [21] where the increased HR was related to increased SiS.

These results must be interpreted with caution because of some limitations of the study. In particular, the small sample size, unequal distribution across gender and age, and the rather short duration of the VR game. Furthermore, the study design did not include familiarization scenario which could help some participants to get acquainted with the controllers.

\section{Conclusion}

The results presented in this paper support findings in previous research on the observation that a free teleportation technique induces less SiS. Moreover, the results may help to understand the SiS induced by locomotion in VR better. A VR gaming environment with minimal induced discomfort could be a powerful user evaluation tool in the field of HCI.

Considering the findings, a future study might include a different locomotion technique, such as rapid, continuous movement between nodes, and different VR game scenarios. The occurrence of no visible difference in the physiological signals points to larger and diverse sample size and longer exposure time. Further investigation is needed to estimate the influence of locomotion techniques to SiS outbreak in VR games.

\section{References}

1. Allen, J.: Photoplethysmography and its application in clinical physiological measurement. Physiological measurement 28(3), R1 (2007)

2. Bowman, D.A., Koller, D., Hodges, L.F.: Travel in immersive virtual environments: An evaluation of viewpoint motion control techniques. In: Virtual Reality Annual International Symposium, 1997., IEEE 1997. pp. 45-52. IEEE (1997)

3. Bozgeyikli, E., Raij, A., Katkoori, S., Dubey, R.: Point \& teleport locomotion technique for virtual reality. In: Proceedings of the 2016 Annual Symposium on Computer-Human Interaction in Play. pp. 205-216. ACM (2016)

4. Bruder, G., Lubas, P., Steinicke, F.: Cognitive resource demands of redirected walking. IEEE transactions on visualization and computer graphics 21(4), 539544 (2015)

5. Carreiras, C., Alves, A.P., Lourenço, A., Canento, F., Silva, H., Fred, A., et al.: BioSPPy: Biosignal processing in Python (2015-), https://github.com/PIAGroup/BioSPPy/

6. Cobb, S.V., Nichols, S., Ramsey, A., Wilson, J.R.: Virtual reality-induced symptoms and effects (vrise). Presence: Teleoperators \& Virtual Environments 8(2), 169-186 (1999) 
7. Darken, R.P., Cockayne, W.R., Carmein, D.: The omni-directional treadmill: a locomotion device for virtual worlds. In: Proceedings of the 10th annual ACM symposium on User interface software and technology. pp. 213-221. ACM (1997)

8. Edeker, A.M., Syomik, A.P., Siyanko, A.: Networked computer system for communicating and operating in a virtual reality environment (Sep 11 2007), uS Patent $7,269,632$

9. Frommel, J., Sonntag, S., Weber, M.: Effects of controller-based locomotion on player experience in a virtual reality exploration game. In: Proceedings of the 12 th International Conference on the Foundations of Digital Games. p. 30. ACM (2017)

10. Habgood, J., Moore, D., Wilson, D., Alapont, S.: Rapid, continuous movement between nodes as an accessible virtual reality locomotion technique. 2018 IEEE Conference on Virtual Reality and 3D User Interfaces (VR) pp. 371-378 (2018)

11. Huang, J.Y.: An omnidirectional stroll-based virtual reality interface and its application on overhead crane training. IEEE Transactions on Multimedia 5(1), 39-51 (2003)

12. Jinjakam, C., Hamamoto, K.: Simulator sickness in immersive virtual environment. In: Biomedical Engineering International Conference (BMEiCON), 2012. pp. 1-4. IEEE (2012)

13. Kennedy, R.S., Lane, N.E., Berbaum, K.S., Lilienthal, M.G.: Simulator sickness questionnaire: An enhanced method for quantifying simulator sickness. The international journal of aviation psychology 3(3), 203-220 (1993)

14. Kennedy, R.S., Lane, N.E., Lilienthal, M.G., Berbaum, K.S., Hettinger, L.J.: Profile analysis of simulator sickness symptoms: Application to virtual environment systems. Presence: Teleoperators \& Virtual Environments 1(3), 295-301 (1992)

15. Kennedy, R., Berbaum, K., Lilienthal, M., Dunlap, W., Mulligan, B.: Guidelines for alleviation of simulator sickness symptomatology. Tech. rep., NAVAL TRAINING SYSTEMS CENTER ORLANDO FL (1987)

16. Kim, Y.Y., Kim, H.J., Kim, E.N., Ko, H.D., Kim, H.T.: Characteristic changes in the physiological components of cybersickness. Psychophysiology 42(5), 616-625 (2005)

17. Lin, J.W., Duh, H.B.L., Parker, D.E., Abi-Rached, H., Furness, T.A.: Effects of field of view on presence, enjoyment, memory, and simulator sickness in a virtual environment. In: Virtual Reality, 2002. Proceedings. IEEE. pp. 164-171. IEEE (2002)

18. McMahan, R.P., Bowman, D.A., Zielinski, D.J., Brady, R.B.: Evaluating display fidelity and interaction fidelity in a virtual reality game. IEEE Transactions on Visualization \& Computer Graphics (4), 626-633 (2012)

19. Min, B.C., Chung, S.C., Min, Y.K., Sakamoto, K.: Psychophysiological evaluation of simulator sickness evoked by a graphic simulator. Applied ergonomics 35(6), 549-556 (2004)

20. Mousavi, M., Jen, Y.H., Musa, S.N.B.: A review on cybersickness and usability in virtual environments. In: Advanced Engineering Forum. vol. 10, pp. 34-39. Trans Tech Publications Ltd. (2013)

21. Nalivaiko, E., Davis, S.L., Blackmore, K.L., Vakulin, A., Nesbitt, K.V.: Cybersickness provoked by head-mounted display affects cutaneous vascular tone, heart rate and reaction time. Physiology \& behavior 151, 583-590 (2015)

22. Nichols, S., Haldane, C., Wilson, J.R.: Measurement of presence and its consequences in virtual environments. International Journal of Human-Computer Studies 52(3), 471-491 (2000) 
23. Peper, E., Harvey, R., Lin, I.M., Tylova, H., Moss, D.: Is there more to blood volume pulse than heart rate variability, respiratory sinus arrhythmia, and cardiorespiratory synchrony? Biofeedback 35(2) (2007)

24. Razzaque, S., Kohn, Z., Whitton, M.C.: Redirected walking. In: Proceedings of EUROGRAPHICS. vol. 9, pp. 105-106. Citeseer (2001)

25. Reason, J.T., Brand, J.J.: Motion sickness. Academic press (1975)

26. Riccio, G.E., Stoffregen, T.A.: An ecological theory of motion sickness and postural instability. Ecological psychology 3(3), 195-240 (1991)

27. wireless biosignals S.A., P.: Plux research kit (2018), http://www.biosignalsplux.com/

28. Schubert, T., Friedmann, F., Regenbrecht, H.: The experience of presence: Factor analytic insights. Presence: Teleoperators \& Virtual Environments 10(3), 266-281 (2001)

29. Slater, M., Usoh, M., Steed, A.: Taking steps: the influence of a walking technique on presence in virtual reality. ACM Transactions on Computer-Human Interaction (TOCHI) 2(3), 201-219 (1995)

30. Souman, J.L., Giordano, P.R., Schwaiger, M., Frissen, I., Thümmel, T., Ulbrich, H., Luca, A.D., Bülthoff, H.H., Ernst, M.O.: Cyberwalk: Enabling unconstrained omnidirectional walking through virtual environments. ACM Transactions on Applied Perception (TAP) 8(4), 25 (2011)

31. Sra, M., Xu, X., Mottelson, A., Maes, P.: Vmotion: Designing a seamless walking experience in vr. In: Proceedings of the 2018 on Designing Interactive Systems Conference 2018. pp. 59-70. ACM (2018)

32. Steinicke, F., Bruder, G., Jerald, J., Frenz, H., Lappe, M.: Analyses of human sensitivity to redirected walking. In: Proceedings of the 2008 ACM symposium on Virtual reality software and technology. pp. 149-156. ACM (2008)

33. Tarvainen, M.P., Niskanen, J.P., Lipponen, J.A., Ranta-Aho, P.O., Karjalainen, P.A.: Kubios hrv-heart rate variability analysis software. Computer methods and programs in biomedicine 113(1), 210-220 (2014)

34. Telford, A., Malpeli, R., Whittle, R., Seery, P., Corrie, M.: Physical Education: VCE Units 1 \& 2. Thomas Nelson Australia (2017)

35. Templeman, J.N., Denbrook, P.S., Sibert, L.E.: Virtual locomotion: Walking in place through virtual environments. Presence 8(6), 598-617 (1999)

36. Terziman, L., Marchal, M., Emily, M., Multon, F., Arnaldi, B., Lécuyer, A.: Shakeyour-head: Revisiting walking-in-place for desktop virtual reality. In: Proceedings of the 17th ACM Symposium on Virtual Reality Software and Technology. pp. 27-34. ACM (2010)

37. Uliano, K., Lambert, E., Kennedy, R., Sheppard, D.: The effects of asynchronous visual delays on simulator flight performance and the development of simulator sickness symptomatology. Tech. rep., ESSEX CORP ORLANDO FL (1986)

38. Wagner, J., Lingenfelser, F., Baur, T., Damian, I., Kistler, F., André, E.: The social signal interpretation (ssi) framework: multimodal signal processing and recognition in real-time. In: Proceedings of the 21st ACM international conference on Multimedia. pp. 831-834. ACM (2013)

39. Walch, M., Frommel, J., Rogers, K., Schüssel, F., Hock, P., Dobbelstein, D., Weber, M.: Evaluating vr driving simulation from a player experience perspective. In: Proceedings of the 2017 CHI Conference Extended Abstracts on Human Factors in Computing Systems. pp. 2982-2989. ACM (2017)

40. Wiederhold, B.K., Davis, R., Wiederhold, M.D.: The effects of immersiveness on physiology. (1998) 
41. Williams, B., Bailey, S., Narasimham, G., Li, M., Bodenheimer, B.: Evaluation of walking in place on a wii balance board to explore a virtual environment. ACM Transactions on Applied Perception (TAP) 8(3), 19 (2011)

42. Witmer, B.G., Singer, M.J.: Measuring presence in virtual environments: A presence questionnaire. Presence 7(3), 225-240 (1998) 